\title{
Hacia una didáctica de los Archivos Escolares: Apuntes para su posicionamiento en las comunidades educativas desde la Formación Ciudadana
}

\author{
Towards a didactic of the School Archives: \\ Notes for its positioning in educational communities \\ from Citizen Education \\ Carla Mella Barrientos \\ Universidad Bernardo O`Higgins-CHILE \\ Liceo de Aplicación \\ carla.mella.barrientos@gmail.com \\ Roberto Rojas Cisterna \\ Universidad Bernardo O`Higgins-CHILE \\ Liceo de Aplicación \\ robertorc31@hotmail.com
}

\section{Resumen}

La creación de Archivos Escolares pensados desde la Formación Ciudadana representa la urgente necesidad de posicionar a estos dispositivos como elementos de memoria e identidad presentes en cada institución educativa. Dicha naturaleza permite comprender tanto las diversas posibilidades en el trabajo docente como las oportunidades para el desarrollo de actividades educativas, que configuran estos espacios como instancias válidas dentro del curriculum escolar y la construcción de ciudadanía de forma transversal. Desde de una experiencia práctica en un Archivo Escolar con estudiantes secundarios, el presente artículo propone algunos lineamientos para implementarlo, junto con el desarrollo de una propuesta de modelo didáctico que posiciona a las y los estudiantes como protagonistas de su proceso de aprendizaje-enseñanza.

Palabras clave: Archivos Escolares, Formación Ciudadana, Didáctica.

\begin{abstract}
The creation of School Archives based on Citizen Education represents the urgent need to position these devices as elements of memory and identity present in every educational establishment. This nature enables us to understand the multiple possibilities in the teaching work as well as the opportunities for the development of educational activities, that shape these spaces as valid requests within the school curriculum and the construction of citizenship on a transversal basis. From a practical experience in a School Archive with high school students, this article proposes some guidelines to implement it, along with the development of a teaching model proposal that positions students as protagonists of its own
\end{abstract}


teaching-learning process.

Keywords: School Archives, Citizen Education, Didactic.

Recibido: 20 de noviembre de 2020 - Aceptado: 18 de enero de 2021

\section{El problema didáctico en los Archivos Escolares}

Nosotros -tanto educadores como ciudadanos- debemos decidir cuál tendría que ser el propósito de esta Educación para la Ciudadanía. Esto significa preguntarnos en qué tipo de sociedad y de mundo queremos vivir, y realizar acciones para convertir esta idea en realidad. Y, de manera particular, cómo queremos que sea nuestra sociedad democrática. Con el objetivo de construir un significado para la Educación Cívica y para la Ciudadanía, debemos preguntarnos estas cuestiones no únicamente desde un punto de vista abstracto o retórico, sino vinculándolo a las experiencias diarias y a nuestras prácticas profesionales como maestros y profesores

(Ross y Vinson, 2012: 73).

El proceso de levantamiento y co-construcción de archivos en instituciones educativas comprende trayectorias no exentas de grandes conflictos, debido a la falta de herramientas teóricas y/o desconocimiento de metodologías por parte de los propios actores escolares. Esta situación, muy lejos de entenderse como un aspecto negativo, se debe comprender como una posibilidad para el desarrollo de prácticas pedagógicas vinculadas a la Formación Ciudadana dentro de las comunidades educativas, lo que da importancia a la necesidad de comprender a los archivos como dispositivos de memoria e identidad para las instituciones que los custodian y los sujetos que se encargan de su mantenimiento y proyección en el tiempo.

El desarrollo de iniciativas de carácter archivístico en espacios escolares responde a preocupaciones que emanan, principalmente, de docentes cuyos intereses se aprestan a la conservación de la memoria histórica de cada establecimiento educativo. Este objetivo adquiere diferentes matices dependiendo de los registros presentes y conservados en cada institución, obligando a los profesores interesados a definir estrategias de trabajo sobre las condiciones materiales presentes. Los mecanismos de acción pueden variar entre una institución escolar y otra, considerando las posibilidades que entregan la gestión y administración educativa en cada territorio.

A partir de este panorama y desde la experiencia de trabajo archivístico en una instancia extraprogramática de Educación Patrimonial, con estudiantes secundarios del Liceo de Aplicación de Santiago de Chile, nos encontramos con decisiones que nos invitan a re-pensar y cuestionar 
la(s) función(es) de los Archivos Escolares (en adelante AE) y la vinculación real con sus comunidades, contribuyendo a los debates actuales sobre el derecho de acceso, la democratización de los espacios archivísticos, el rol político de los sujetos encargados de los archivos, las políticas de adquisición y donación, etc. Este cambio de paradigma, sin duda, es parte de una larga trayectoria que incluye perspectivas que coexisten dentro del panorama archivístico actual, y que obligan a cuestionar las funciones materiales del Archivo, tanto de contenido como su vinculación con la sociedad, además de las metodologías de trabajo encargadas para su co-construcción y utilización como dispositivo didáctico.

El objetivo de este artículo es definir el concepto de Archivo Escolar, plantear argumentos para su levantamiento y establecer los principales lineamientos de un modelo de trayecto didáctico con un enfoque de Formación Ciudadana, a partir del estudio del trabajo realizado con estudiantes secundarios del Taller Patrimonial del Liceo de Aplicación en el período 2018-2019. Para el proceso de recolección de evidencias de aprendizaje que permitan sustentar la propuesta, se desarrolló un focus group con los estudiantes involucrados en el trabajo de AE que nos permitió visibilizar desde sus percepciones, los aprendizajes en clave ciudadana y los elementos procedimentales que sustentan la propuesta desde la siguiente pregunta guía: ¿Qué elementos resignifican el concepto de AE desde una propuesta de levantamiento y trabajo en perspectiva de Formación Ciudadana a partir de una experiencia de trabajo con estudiantes secundarios en un taller de Educación Patrimonial?
El problema de estudio podemos definirlo como una aproximación didáctica para el sustento pedagógico y curricular desde la Educación Patrimonial, en el contexto del auge de AE, en tanto la importancia de su levantamiento y su utilización como recurso educativo con una perspectiva ciudadana desde y para las comunidades educativas.

\section{Del Archivo Histórico al Archivo Escolar con perspectiva ciudadana}

El concepto de Archivo posee larga data de definiciones y límites en cuanto a su uso. Para efectos de este artículo, se lo reconocerá desde tres vertientes. La primera de ellas es la influencia de Elio Lodolini, quien califica como igual de importante al conjunto de documentos que componen un archivo y las relaciones existentes entre ellos desde su origen (1995: 42), es decir, plantea el sentido y valor del contexto al momento de levantar un Archivo documental, calificándolo como un asiento de memoria que nace espontáneamente “como sedimentación documental de una actividad práctica, administrativa, jurídica” (1993: 24). Esta contribución resulta de gran importancia para este estudio, ya que invita a reconocer el origen de los documentos en tanto contexto de producción asumiendo, además, que no toda recopilación o colección de documentos pueden ser efectivamente un Archivo pues en este caso, solo cumpliría con las características presentes en los almacenes de papeles.

En segundo lugar, se encuentra Antonia Heredia (1991) quien considera relevante la apertura del Archivo a la sociedad, a través del denominado "servicio". Ella fue quién definió al Archivo como: 
uno o más conjuntos de documentos, sea cual sea su fecha, su forma y soporte material, acumulados en un proceso natural por una persona o institución pública o privada en el transcurso de su gestión conservados, respetando aquel orden, para servir como testimonio e información para la persona o institución que los produce, para los ciudadanos o para servir de fuentes de historia (89).

Reconociendo el rol académico-historiográfico que adquiere el Archivo respecto a instituciones con funciones y actividades. A pesar de integrar una perspectiva aun tradicional sobre la definición de Archivo basada en el "orden natural" y como documentos que "prueban, justifican y ofrecen información de las actividades propias del productor de carácter clasificado y seriado", Heredia pone de manifiesto el carácter social que alcanzan los documentos, y particularmente el Archivo, para los ciudadanos de un país, los llamados "beneficiarios" de su resguardo y conservación.

En tercer lugar, se encuentra la transformación progresiva del sentido archivístico que dio cuerpo al denominado "paradigma post-custodial" que centra el debate en el rol "político" del Archivo. Uno de sus principales exponentes es el inglés Terry Cook (2010), quien puso a prueba las definiciones tradicionales que se enfocan en la objetividad del Archivo, cuestionando su pasividad y neutralidad. Esta contribución reside principalmente en reconocer a los sujetos que componen el archivo, los llamados "co-creadores" y la re-definición de la función del Archivo. Tal es el caso del Archivo y los Archivos, que para el autor se perciben, desde la singularidad y la pluralidad, como "un discurso, una metáfora, un símbolo o una manifestación de poder, como un sitio de inscripción e intencionalidad humana y de memoria cuestionada" (153-154) y una realidad puesta en permanente tensión, respectivamente. Es decir, rescata la subjetividad de los archiveros quienes desarrollan un trabajo cruzado por sus "propias suposiciones, creencias, teorías, estrategias, metodologías y procedimientos profesionales y disciplinarios que continuamente dan forma a la naturaleza del documento archivístico y de los archivos" (153-154) y que además, se encuentran de manera permanente ejecutando actividades en instituciones que se vinculan con territorios y realidades en donde ejercen una visión particular del mundo.

Es precisamente en este contexto de cambio de paradigmas respecto al concepto de Archivo, que surge una nueva tipología que son los AE. Muchos de los Archivos que nacieron en establecimientos educativos surgieron como Archivos Históricos Escolares dado su origen involuntario y cercano al rescate de la actividad administrativa y de gestión; sin embargo, en el último tiempo es posible reconocer la convivencia entre este tipo de iniciativas prematuras y la potencialidad creativa e investigativa de los talleres para estudiantes que han acompañado y desarrollado actividades de difusión y acceso a estos espacios, dando origen a lo que en esta publicación llamamos "Archivo Escolar".

La conformación de los AE en Chile surgió a partir de iniciativas que impulsaron el trabajo con Archivos en las comunidades escolares, como el Programa de Archivos Escolares (PAE-UC) liderado 
por la Universidad Católica de Chile; la apertura de planes de perfeccionamiento profesional, como el Taller de Archivística Comunitaria (TAC) organizado por el Archivo de la Federación de Estudiantes de la Universidad de Chile; además de Diplomados de postítulo, y el significativo trabajo voluntario de profesores y profesionales de las Humanidades y Ciencias Sociales. Bajo estas condiciones de posibilidad, surgieron los primeros $\mathrm{AE}$ en el país, principalmente a partir del rescate del acervo documental creado de forma involuntaria por algunas instituciones educativas y visibilizados a través del reconocimiento del valor en tanto patrimonio y memoria.

En este artículo, el AE es definido como un dispositivo político, culturaly didáctico en permanente construcción, cuestionamiento y colaboración. Por su naturaleza, se encuentra en constante tensión con la identidad institucional, lo que también permite que sea una iniciativa que surge desde los sujetos pertenecientes a una comunidad educativa en particular, quienes también son encargados de representarla, problematizando el pasado a partir de los desafíos del presente. El AE se constituye como un espacio físico y/o digital cuyo contenido es el fruto de una deliberación crítica de los archiveros/as a cargo, acerca de qué se resguarda, cómo se da acceso y a quiénes se difunden la documentación, la cual se encuentra en diversos soportes y materialidades, dando cuenta de la impronta de la comunidad educativa y su trayectoria en tanto espacio-tiempo.

En consecuencia, los AE se crean voluntariamente y se evidencian desde su potencialidad democratizadora respecto a las posibilidades de mediación con el pasado "en medio del cuestionamiento generalizado a los medios de transmisión intergeneracional del conocimiento, como los modelos tradicionales de aprendizaje centrados en la escuela" (Bianculli et al., 2018: 5). Es decir, cada organización tiene el "control sobre las prácticas de documentación” (Andaur, 2015: 224-225), como su ordenación y clasificación, evidenciando en este proceso los "aspectos de la identidad o de interés de la comunidad que lo creó" (Andaur, 2015: 224-225), el acceso abierto o semi-abierto a los documentos que integran el Archivo y, la participación permanente de su comunidad.

Siguiendo la línea de Rodrigo Mayorga, María José Vialy Rodrigo Sandoval(2019) la creación de estas instancias de producción de conocimiento debe ser garantizada de manera efectiva, no limitándose al espacio académico, sino al territorio que la circunda, abriendo espacios de trabajo colectivo con otros actores y comunidades educativas. Las comunidades productoras son especialmente relevantes dentro de la definición de AE que se plantea en este artículo, dado que nos entregan en el presente la posibilidad de valorar altamente la documentación producida y conservada dentro de cada institución escolar. En definitiva, los

[...] archivos son prueba y memoria a la vez, son huella documental e instrumento para el ejercicio de derechos: aquel que me vincula con el presente y que permite el desenvolvimiento de una institución y aquel que permite que auscultemos las decisiones que hemos tomado (Mayorga et al., 2019: 5-6). 
Uno de los actores colectivos más importantes dentro del trabajo con AE son los/as docentes de los establecimientos escolares, quienes cuentan con un rol de mediación activa frente a los recursos entregados por estos espacios de memoria y patrimonio. Es destacable la serie de ventajas presentes en el trabajo con el AE, ya sea desde un punto de vista archivístico como lo es la ordenación, clasificación y conservación; o desde lo pedagógico, generando nuevas estrategias didácticas que den cuenta de un sentido crítico o bien en el uso del archivo como herramienta para la investigación, interpretación, explicación y reconocimiento de sus acervos documentales por parte de los estudiantes y sus comunidades.

El uso del Archivo en esta perspectiva permite redefinir las dinámicas presentes en el aula, transformando los paradigmas pedagógicos y educativos. Este cambio supone

[...] pasar de un modelo educativo centrado en la transmisión pasiva de conocimientos a uno focalizado en la adquisición de habilidades propias del pensamiento crítico y la comunicación. Un modelo educativo menos centrado en evaluaciones estandarizadas y más en lo que nuestros estudiantes son capaces de descubrir, crear y comunicar respecto al mundo que los rodea (Mayorga et al., 2019: 6).

Bajo esta experiencia, el AE se transforma en un foco de interpelación constante respecto a la Formación Ciudadana, desde un punto de vista marcado por los estudiantes como constructores de conocimiento y los profesores como mediadores activos, sujetos que en su conjunto trabajan por el rescate de la memoria y el patrimonio dentro de instituciones educativas con una historia viva y dinámica.

Desde lo metodológico, el trabajo de pensamiento crítico realizado en los $\mathrm{AE}$, se vincula con la Formación Ciudadana, dado que colabora con la comunidad próxima y tiene un impacto desde un ámbito socio-político, posicionándose como un principio rector transversal dado que

La formación ciudadana no se agota en la introducción de un curso de alfabetización jurídica para los estudiantes, ni se resuelve con un plan de formación ciudadana para escuela de manera particular. Es urgente ampliar la mirada planteándonos cuestiones fundamentales sobre la sociedad de la que somos parte (Bravo, 2016:74)

En ese sentido, la vinculación de los desafíos del presente con la búsqueda de respuesta en las fuentes de los $\mathrm{AE}$, nos posiciona desde una perspectiva de una ciudadanía democrática crítica donde el conflicto es eje central (Jiménez et al., 2013), el cual emerge al contrastar y "discutir" con la documentación, lo que se configura como un ejercicio político dentro de los espacios de aula (Rossy Vinson, 2012), posibilitando desarrollo de temáticas desde tópicos ciudadanos transversales de la Formación Ciudadana en espacios escolares, tales como Derechos Humanos, responsabilidad social, problemas sociales, consensos y disensos, valor de lo público, convivencia social, conflicto, cuestión política, mecanismos y niveles de participación, toma de decisiones, etc. (Jiménez, 2017). 
Con esta óptica, el levantamiento y trabajo didáctico con fuentes de $\mathrm{AE}$, permite reconstruir la historicidad de sus actores con eje central en rol de los estudiantes-ciudadanos/as. Su creación, por tanto, resulta un acto de memoria y rescate frente a la conformación histórica de una cultura del desecho material y el escaso valor ante los registros documentales presentes. Por tanto,

Que una institución de enseñanza tome la decisión de iniciar un proceso de reconstrucción y preservación de la memoria, incide sin lugar a dudas en quienes trabajan en ella y sobre todo en sus estudiantes, en las nuevas generaciones. Sensibilizar y movilizar lo relativo al resguardo de la historia, significa apropiarse y resignificar trayectorias e identidades (Murrielo et al., 2011: 1).

Esta perspectiva de los AE como posibilidad de articular procesos transversales de construcción ciudadana en espacios educativos, busca convertirse en una posibilidad más de democratización, acceso a la información y desarrollo de aprendizajes comunitarios para las y los estudiante, en co-construcción con sus docentes.

\section{Planteamiento metodológico}

La metodología para levantar esta propuesta se plantea bajo un carácter exploratorio, basada en la experiencia de trabajo dentro del AE del Liceo de Aplicación en Santiago de Chile y las reflexiones surgidas en el Taller de Archivística Comunitaria del Archivo FECH (2019). Estos insumos permiten reconocer desdelo teórico las principales corrientes que lo diferencian de un Archivo Histórico tradicional, y a su vez, plantear desde la práctica los principales desafíos, los pasos elementales para su levantamiento desde la Formación Ciudadana y sus posibilidades didácticas.

En cuanto al diseño de la investigación, se posiciona desde lo cualitativo, en tanto tiene como objetivo sistematizar en un modelo didáctico la experiencia de trabajo archivístico con estudiantes secundarios. Por lo que inspirados en la metodología de la investigación-acción, buscamos proponer saber pedagógico desde y para las comunidades educativas, en co-construcción con el trabajo de estudiantes secundarios, de forma de empoderar a los mismos actores (Latorre, 2005:12).

Como este estudio didáctico se desarrolló desde una experiencia y actores concretos, se establece como un estudio de casos en clave investigación-acción (Latorre, 2005), por lo que para recoger información e insumos que posibilitaron la sistematización del modelo de trabajo, se identificaron los principales argumentos dados para el levantamiento del Archivo Escolar del Liceo de Aplicación, como se ligó al Plan de Formación Ciudadana y cuáles son sus nexos con las políticas públicas vigentes. Junto a eso, para el desarrollo de los principios del modelo didáctico, se realizó un grupo de enfoque con un grupo de 16 estudiantes secundarios del Liceo de Aplicación pertenecientes al Taller Patrimonial (espacio extra programático), lo que permitió un diálogo e interacción que permitiera construir significados colectivos (Sampieri, et al 2014: 409), a partir de las siguientes tópicos de los que se dialogó: 
1. Relación del patrimonio con las fuentes del AE.

\section{La importancia ciudadana del trabajo de los AE} para la identidad de la comunidad.

3. Metodologías a partir de desarrollo de preguntas problematizadoras.

\section{Principales aprendizajes.}

La conversación con los estudiantes fue grabada en formato mp3 y transcrita íntegramente para luego ser procesada en el software de estudios sociales ATLAS.TI, en el cual pudimos construir una familia, categorías y códigos inspirados en elementos de Teoría Fundamentada, desarrollando una codificación emergente desde los elementos centrales que tenían en común las respuestas de los participantes y que se visibilizaran desde sus propias "voces" a partir de una serie de "barridos" hasta afinar códigos analíticos (Soneira, 2006: 155 - 156), como se muestra a continuación:

\section{Tabla 1: Sistema de codificación de grupo de enfoque}

\begin{tabular}{|c|c|c|c|c|}
\hline Familias & Categorías & Códigos & Definiciones & Frecuencias \\
\hline \multirow[t]{6}{*}{$\begin{array}{l}\text { Elementos } \\
\text { Apropiados }\end{array}$} & \multirow[t]{2}{*}{$\begin{array}{l}\text { Formación } \\
\text { Ciudadana }\end{array}$} & $\begin{array}{l}\text { Ciudadanía Activa } \\
\text { Transformativa }\end{array}$ & $\begin{array}{l}\text { Expresiones que denotan un } \\
\text { compromiso por realizar aportes a } \\
\text { generar cambios en su comunidad } \\
\text { educativa, fomentando acciones para } \\
\text { una mayor conciencia de los distintos } \\
\text { estamentos de formas concretas. }\end{array}$ & 6 \\
\hline & & $\begin{array}{l}\text { Uso del espacio } \\
\text { público }\end{array}$ & $\begin{array}{l}\text { Reacciones que tienen las distintas } \\
\text { personas al observar, participar e } \\
\text { involucrarse con las intervenciones y/o } \\
\text { acciones de socializar en el espacio } \\
\text { público lo elaborado por los estudiantes. }\end{array}$ & 6 \\
\hline & \multirow[t]{2}{*}{ Identidad } & $\begin{array}{l}\text { Discurso } \\
\text { emergente }\end{array}$ & $\begin{array}{l}\text { Levantar un discurso identitario distinto } \\
\text { al "oficial", de elementos que los } \\
\text { estudiantes consideran que enriquecen } \\
\text { las discusiones de los desafíos del } \\
\text { presente de la comunidad educativa. }\end{array}$ & 8 \\
\hline & & $\begin{array}{l}\text { Recuperación } \\
\text { Memoria } \\
\text { Histórica }\end{array}$ & $\begin{array}{l}\text { Importancia de relevar y la memoria } \\
\text { histórica por parte de los estudiantes, } \\
\text { como un eje esencial en la labor del } \\
\text { Taller en tanto su propia conciencia } \\
\text { histórica como la de su comunidad. }\end{array}$ & 6 \\
\hline & $\begin{array}{l}\text { Diálogo } \\
\text { Presente- } \\
\text { Pasado } \\
\end{array}$ & $\begin{array}{l}\text { Comunidad ayer- } \\
\text { hoy }\end{array}$ & $\begin{array}{l}\text { Problematización de la comunidad } \\
\text { educativa en el pasado y el presente, en } \\
\text { cuanto a las formas de lucha y unión. }\end{array}$ & 4 \\
\hline & $\begin{array}{l}\text { Procedimie } \\
\text { ntos }\end{array}$ & $\begin{array}{l}\text { Preguntas } \\
\text { problematizadoras }\end{array}$ & $\begin{array}{l}\text { Elementos que permitieron elaborar en } \\
\text { forma conjunta por los estudiantes, } \\
\text { preguntas que llamen a la reflexión de } \\
\text { una problemática actual de la } \\
\text { comunidad en perspectiva histórica. }\end{array}$ & 7 \\
\hline
\end{tabular}


Fuente: elaboración propia.

Con esta codificación, se posibilitó la emergencia de principios, conceptos y procedimientos para el establecimiento de nuestra propuesta, realizando a su vez el cruce con los elementos curriculares que sustentan institucionalmente el desarrollo de las actividades de levantamiento, construcción y trabajo en los AE.

\section{Hacia una didáctica de los Archivos Escolares}

Desde el cruce entre lo planteado pedagógicamente en diversas instancias de trabajo con el AE, los argumentos curriculares y los elementos destacados por los estudiantes, se desarrolla la siguiente propuesta en dos líneas: la primera, sobre los argumentos curriculares para su implementación y la segunda, sobre un modelo didáctico para su uso pedagógico.

\subsection{Posicionando los Archivos Escolares desde la Formación Ciudadana}

En el contexto de crisis de legitimidad de las instituciones y autoridades políticas, la Escuela se ha posicionado como un espacio de experiencias de desarrollo de una ciudadanía activa por parte de los/as estudiantes. Más aún con el avance de distintas políticas públicas que apuntan a la Formación Ciudadana con un enfoque transversal en las escuelas chilenas, las que consecutivamente inician de un diagnóstico común sobre las carencias en el conocimiento ciudadano por parte de los/as estudiantes, lo que también ha sido constatado por el Estudio Internacional de Educación Cívica y Formación Ciudadana (ICCS, 2016).
Esta carencia ha intentado ser suplida en parte por la Ley 20.911, que crea los Planes de Formación Ciudadana ( $\mathrm{PFC}$ ), entendiendo que las comunidades escolares deben avanzar a que los/as estudiantes vivan un "proceso formativo continuo que permite que los niños, niñas, jóvenes y adultos desarrollen un conjunto de conocimientos, habilidades y actitudes que resultan fundamentales para la vida en una sociedad democrática" (2016: 1). De esta manera esta ley se posiciona desde los siguientes ejes de acción:

\section{Imagen 1:Dimensiones de la Formación Ciudadana en las Escuelas}

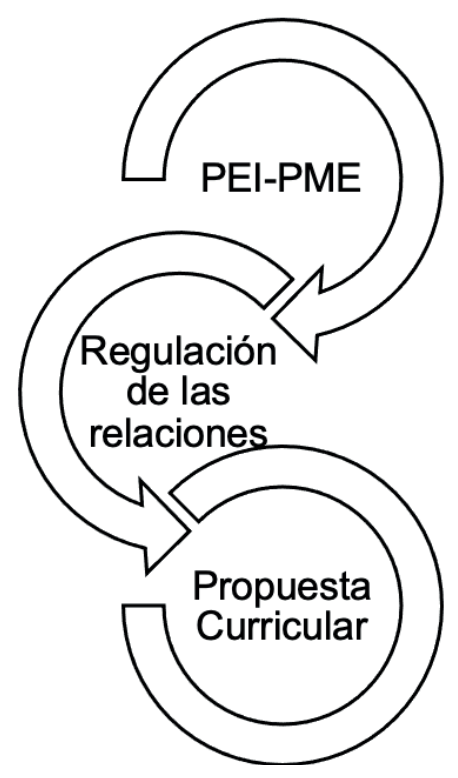

Fuente: Elaborado a partir de principios de Ley 20.911 (2016).

Para los AE, el sustentarlos desde el PEI-PME y como propuesta curricular se conecta con varios de los principios y acciones de la Ley de Formación Ciudadana, tales como: 


\section{Tabla 2: Argumentos desde la Ley de Formación Ciudadana para implementar AE}

\begin{tabular}{|l|l|}
\hline Principios & Acciones concretas propuestas: \\
\hline $\begin{array}{l}\text { b) Fomentar en los estudiantes el ejercicio de una } \\
\text { ciudadanía crítica, responsable, respetuosa, abierta } \\
\text { y creativa. }\end{array}$ & $\begin{array}{l}\text { ii. La realización de talleres y actividades } \\
\text { extraprogramáticas, en los cuales haya una integración } \\
\text { y retroalimentación de la comunidad educativa. }\end{array}$ \\
\hline $\begin{array}{l}\text { f) Fomentar la participación de los estudiantes en } \\
\text { temas de interés público. }\end{array}$ & $\begin{array}{l}\text { iv. El desarrollo de actividades de apertura del } \\
\text { establecimiento a la comunidad. }\end{array}$ \\
\hline $\begin{array}{l}\text { g) Garantizar el desarrollo de una cultura } \\
\text { democrática y ética en la escuela. }\end{array}$ & \\
\hline $\begin{array}{l}\text { h) Fomentar una cultura de la transparencia y la } \\
\text { probidad. }\end{array}$ & \\
\hline
\end{tabular}

Fuente: Ley 20.911 (2016: 1-2)

Estos planteamientos que se interrelacionan, permiten sustentar la importancia de implementar AE como políticas institucionales que puedan ser estables en el tiempo y que configuren una estrategia de construcción identitaria de las comunidades educativas, dado que resignifican la memoria histórica de los distintos actores que conviven en las Escuelas. En consecuencia, se posibilita acceder a horas para docentes e insumos materiales de trabajo para materializar actividades que confluyan en el levantamiento, trabajo y mantenimiento de AE desde y para sus comunidades educativas gracias a la integración de los instrumentos de gestión y mejora pedagógica.

En cuanto al tipo de iniciativas concretas implementadas con el impulso de la ley de Formación Ciudadana en las escuelas, Zúñiga y Cárdenas (2019) señalan que un 34\% corresponde a actos, celebraciones, ferias y conmemoraciones, un $29 \%$ a talleres, un $28 \%$ acciones orientadas al Centro de Estudiantes, un $28 \%$ a salidas pedagógicas, entre otras. En este sentido, no se concibe que actividades como los AE posibilitan un abordaje desde múltiples enfoques, en tanto actividad extraprogramática y como acciones didácticas curriculares.

Es en este contexto, visualizar la implementación de proyectos de AE, ya sea de forma física, virtual o mixta, como una línea de acción para el desarrollo de actividades, principios y posibilidades didácticas para la Formación Ciudadana en las escuelas permite levantar colaborativamente un espacio entre docentes y estudiantes como proceso de autoconocimiento de sus comunidades e indicador de iniciativas innovadoras que generan identidad en el tiempo.

Otro de los elementos a tener en cuenta a la hora de concebir los $\mathrm{AE}$, es la importancia que tiene el acceso a la información como un derecho social. En ese sentido, las comunidades educativas deben fomentar y desarrollar "un conjunto de conocimientos, habilidades y actitudes que resultan fundamentales para la vida en una sociedad democrática" (Mineduc, 2016: 11). Lo que se complementa con otro de los argumentos desde las políticas públicas como es la Ley 20.529 (2011) de Aseguramiento de la Calidad, ya que tiene como 
principio el carácter integral de la educación y el respeto a los DD.HH, posicionando al estudiantado en una sociedad que ejerce el derecho a la información para la toma de decisiones. Cabe destacar que entendemos este derecho no desde la lógica de consumidor, sino que desde la de productor comunitario, dado que la decisión de qué y para qué conservamos, es una disyuntiva eminentemente política y empodera a las comunidades en cuanto a re apropiarse de su memoria histórica.

\section{Imagen 2: Ejemplo de Archivo Escolar físico.}

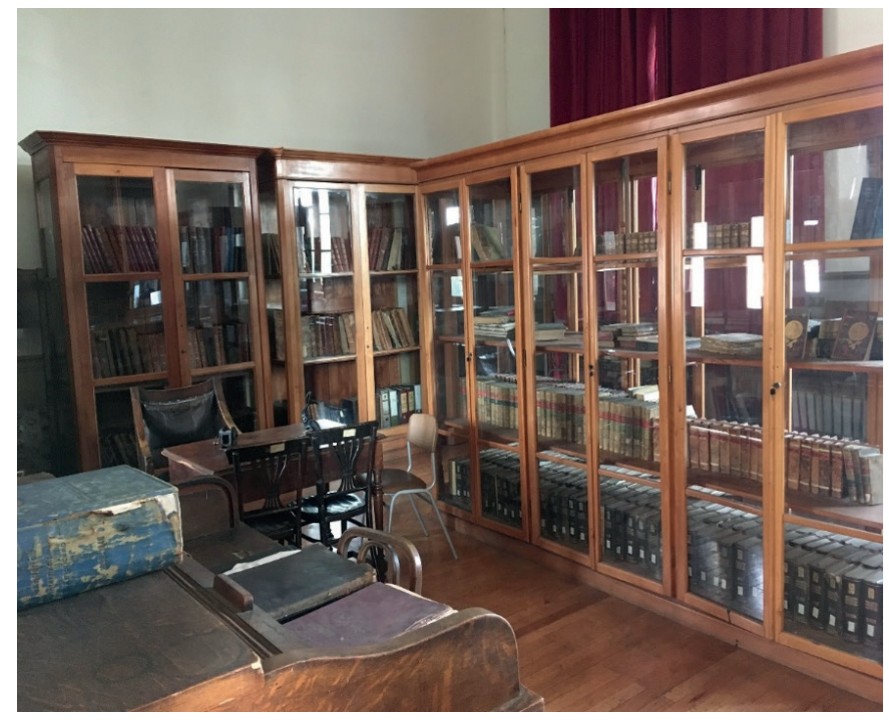

Fuente: Fotografía de los autores, 2017.

El ejemplo del Archivo Escolar del Liceo de Aplicación (en instagram: @patrimonio.ldea) inaugurado a finales de 2015, ha sido una experiencia de conservación, investigación y difusión del patrimonio comunitario e instancia de desarrollo de actividades extra programáticas con estudiantes que realizan investigación para el desarrollo de pensamiento histórico y ciudadano.
Respecto a los argumentos curriculares desde asignaturas concretas en las cuales levantar trayectos desde la didáctica de los Archivos Escolares, podemos señalar las siguientes asignaturas con sus objetivos respectivos:

\section{Tabla 3: Lineamientos curriculares Educación Ciudadana $\left(3^{\circ}\right.$ y $4^{\circ}$ medio)}

Fuente: Mineduc (2020a y 2020b).

En estas asignaturas, se posibilita un nexo muy claro entre historia reciente, DD.HH, problemas socialmente relevantes que desde las comunidades escolares se pueden documentar el cómo se vivieron hechos o procesos a nivel local, con sus respectivas perspectivas y consecuencias. Por este motivo que los $\mathrm{AE}$, en tanto proyectos comunitarios, permiten ser trabajados y estructurados en distintas fases las que pueden tener una duración de un año académico y que tienen una constante proyección en las diversas generaciones al incorporar distintas fuentes y fondos.

Respecto al énfasis curricular en la investigación como habilidad transversal, podemos estructurar objetivos de aprendizajes que lleven a desarrollar la conciencia de problemas actuales que poseen raigambre histórica, los AE posibilitan la problematización en la recopilación de un conjunto de fuentes históricas tales como: revistas, fotografías, panfletos, cuadernos, entrevistas, registros audiovisuales, actas, etc. Trabajándolas como se indicará en el siguiente apartado. 
Tabla 3: Lineamientos curriculares Educación Ciudadana ( $3^{\circ}$ y $4^{\circ}$ medio)

\begin{tabular}{|c|c|c|}
\hline \multicolumn{3}{|l|}{ Objetivos de aprendizaje } \\
\hline Conocimiento y Comprensión & Habilidades & Actitudes Siglo XXI \\
\hline $\begin{array}{l}\text { Educación Ciudadana } \\
\text { OA 06 Reflexionar personal y } \\
\text { grupalmente sobre diversas } \\
\text { formas de participación y su } \\
\text { aporte al fortalecimiento del bien } \\
\text { común, considerando } \\
\text { experiencias } \\
\text { fenómenos personales, } \\
\text { contemporáneos y las } \\
\text { perspectivas del republicanismo, } \\
\text { el liberalismo, y } \\
\text { comunitarismo. }\end{array}$ & $\begin{array}{l}\text { OAH a Investigar sobre la } \\
\text { realidad considerando: } \\
\text { formulación de preguntas o } \\
\text { problemas de investigación a } \\
\text { partir de la observación de } \\
\text { fenómenos; - levantamiento de } \\
\text { información a partir de métodos y } \\
\text { técnicas propias de Historia, } \\
\text { Geografía, Economía y otras } \\
\text { ciencias sociales; - análisis crítico } \\
\text { de las evidencias y evaluación de } \\
\text { su validez, considerando su uso } \\
\text { ético para respaldar opiniones; - } \\
\text { definición del marco teórico, del } \\
\text { estado de la cuestión y de los } \\
\text { conceptos disciplinares del tema a } \\
\text { investigar; - análisis de las propias } \\
\text { conclusiones en relación con los } \\
\text { supuestos iniciales. }\end{array}$ & $\begin{array}{l}\text { OAA 13 Interesarse por las } \\
\text { posibilidades que ofrece la } \\
\text { tecnología para el desarrollo } \\
\text { intelectual, personal y social del } \\
\text { individuo. } \\
\text { OAA 14 Valorar las TIC como } \\
\text { una oportunidad para informarse, } \\
\text { investigar, socializar, } \\
\text { comunicarse y participar como } \\
\text { ciudadano. } \\
\text { OAA 16 Actuar de acuerdo con } \\
\text { los principios de la ética en el uso } \\
\text { de la información y de la } \\
\text { tecnología, respetando la } \\
\text { propiedad intelectual y la } \\
\text { privacidad de las personas }\end{array}$ \\
\hline $\begin{array}{l}\text { Comprensión Histórica del } \\
\text { Presente } \\
\text { OA 05 Participar en el desarrollo } \\
\text { de iniciativas de historia local, } \\
\text { recogiendo relatos y fuentes } \\
\text { propias de la comunidad cercana } \\
\text { para relevar espacios de memoria. }\end{array}$ & $\begin{array}{l}\text { OAH a Investigar sobre la } \\
\text { mentalidad considerando: } \\
\text { Formulación de preguntas o } \\
\text { problemas de investigación a } \\
\text { partir de la observación de } \\
\text { fenómenos; - Levantamiento de } \\
\text { información a partir de métodos y } \\
\text { técnicas propias de historia, } \\
\text { geografia, economía y otras } \\
\text { ciencias sociales; - Análisis crítico } \\
\text { de las evidencias y evaluación de } \\
\text { su validez, considerando su uso } \\
\text { ético para respaldar opiniones; - } \\
\text { Definición del marco teórico, del } \\
\text { estado de la cuestión y de los } \\
\text { conceptos disciplinares del tema } \\
\text { por investigar; - Análisis de las } \\
\text { propias conclusiones en relación } \\
\text { con los supuestos iniciales. }\end{array}$ & $\begin{array}{l}\text { OAA } 08 \text { Trabajar } \\
\text { colaborativamente en la } \\
\text { generación, desarrollo y gestión } \\
\text { de proyectos y la resolución de } \\
\text { problemas, integrando las } \\
\text { diferentes ideas y puntos de vista. }\end{array}$ \\
\hline
\end{tabular}




\subsection{Una propuesta didáctica para el trabajo en Archivos Escolares}

[...] las preguntas que teníamos que hacer no tenían que ser tan directas sino mucho más abierta y el tema de ciudadanía...ciudadanía peligrosa teníamos que tenerlo también en cuenta. Entonces eran muchos factores que cuando hicimos las sesiones anteriores tuvimos que juntarlo en uno solo. Y si no hubiéramos visto eso, como dije antes, el trabajo hubiera sido distinto, hubiera tenido otro enfoque. Es por eso que sí considero que fue necesario tener un modelo teórico que, en mente para poder realizar algo (Estudiante, comunicación personal, 2018).
Un elemento constitutivo de relevancia en este artículo es la importancia de las experiencias de construcción de conocimiento ciudadano y pedagógico desde y para las comunidades escolares, por ende, la difusión de los principios, estrategias y reflexiones acerca del trabajo archivístico y de investigación histórica desde el quehacer cotidiano con estudiantes secundarios tiene por objetivo ser un aporte e insumo a la contextualización y surgimiento de otras nuevas instancias de AE. De esta manera y desde las "voces" de los estudiantes, se grafica de la siguiente forma la codificación que inspiró el modelo a presentar:

\section{Imagen 3: red de códigos del grupo de enfoque con estudiantes participantes}

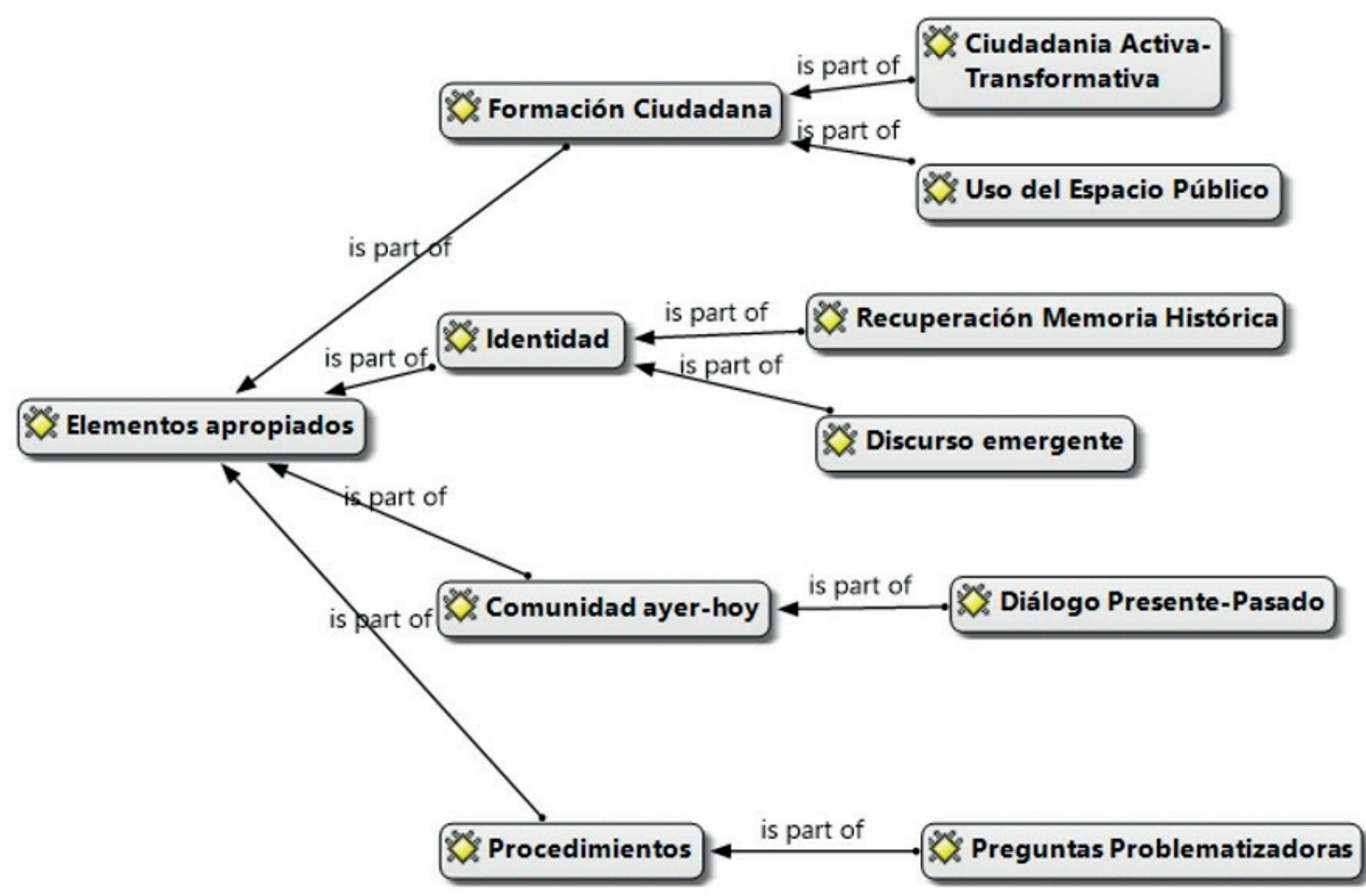


Fuente: elaboración propia en ATLAS.TI.

A partir de la reflexión en torno a lo que ha sido la experiencia del AE del Liceo de Aplicación surgidas en el trabajo con los estudiantes bajo la lógica de ensayo y error desde la Educación Patrimonial, surgió la siguiente pregunta:

¿Existe una didáctica para el trabajo en Archivos Escolares?

Desde la experiencia acumulada en el quehacer pedagógico en el Taller Patrimonial, se puede señalar que sí, efectivamente hay un enfoque didáctico específico que está en constante construcción cuyo fundamento es desarrollar instancias que tengan énfasis en un discurso explicativo-argumentativo, con nudos problemáticos que fomenten un aprendizaje situado y que, a su vez aporte en la construcción de una ciudadanía crítica-transformativa.

Imagen 3: Vinculación Normativa y Didáctica de los AE.

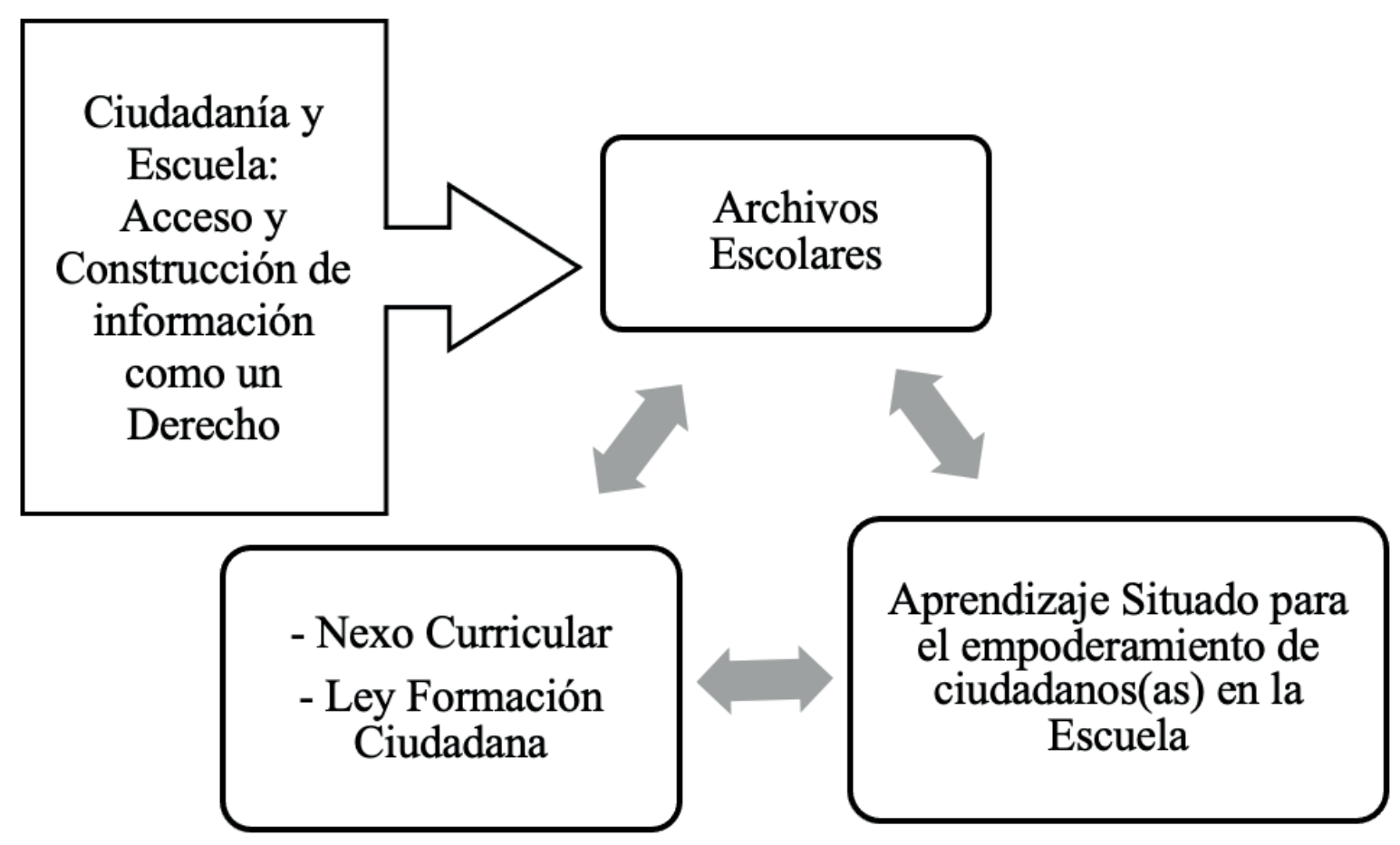


Fuente: elaboración propia.

Esta triangulación sustenta desde lo curricular y normativo, el que docentes puedan levantar propuestas ante los equipos de gestión. Sin olvidar, de manera especial el componente de la didáctica de los $\mathrm{AE}$, el que entendemos cómo se ilustra en la red conceptual siguiente:

\section{Imagen 4: Re-contextualización Archivos} Escolares
Fuente: elaboración propia.

Este diálogo presente-pasado, vinculando lo curricular con los desafíos de las comunidades, permiten la construcción de pensamiento histórico, teniendo como eje a los objetivos de aprendizaje al servicio del desarrollo de una ciudadanía democrática, activa y crítica desde los contenidos, las habilidades y las actitudes que sustentan este posicionamiento. En cuanto a cómo se materializan estos principios didácticos en una secuencia concreta de trabajo con estudiantes, ya sea en

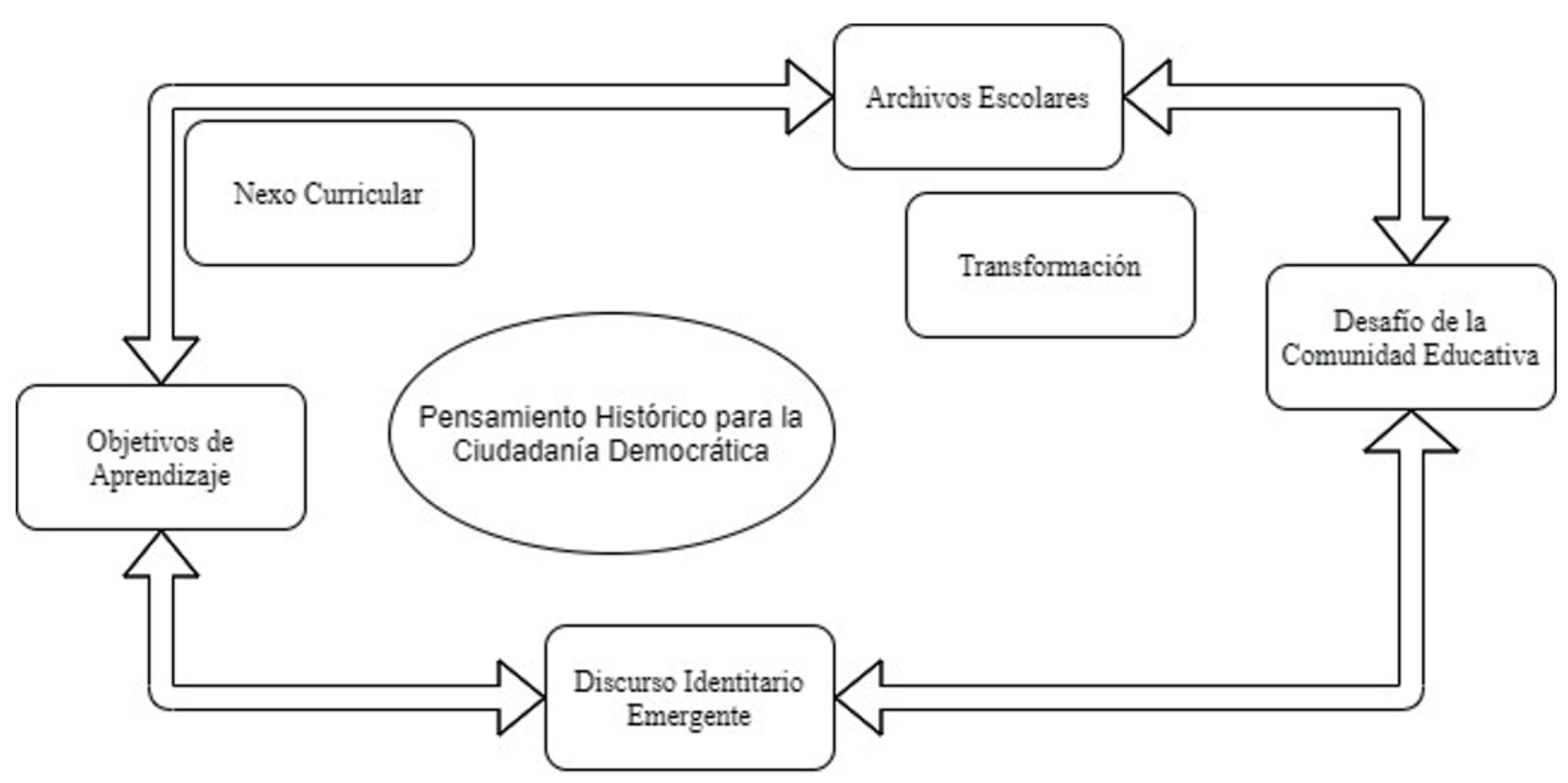

Diálogo Presente Pasado 
asignatura o taller extra programática, la sugerimos desde los elementos centrales presentados a continuación:

\section{Imagen 5: Propuesta trayecto didáctico para trabajo en $\mathrm{AE}$}

Fuente: elaboración propia.

Analizando cada una de las fases del trayecto, podemos señalar a partir del cruce entre las experiencias de enseñanza-aprendizaje realizadas con los estudiantes en las exposiciones e intervenciones realizadas a su comunidad educativa desde el uso de fuentes del AE, lo siguiente:

1.- Problematización desafíos comunitarios del presente: De acuerdo a los intereses y motivaciones de los estudiantes, eligen un tema histórico que les interese a partir de una preocupación desde su presente. Indagan si se ha escrito o producido algo sobre el tema y problematizar qué y por qué les gustaría aprender en el proceso. De esta manera se busca guiar que los estudiantes identifiquen una problemática de su comunidad educativa o contexto territorial, generando así preguntas acerca de las causas históricas de lo que están viviendo en el presente y qué pueden hacer con miras al futuro.

Este cruce con el presente es fundamental, porque permitirá estructurar el trayecto de búsqueda en el AE, indagar fuentes o construirlas (ejemplo entrevistas de historia oral) que puedan ser incorporadas al corpus documental, con el objetivo de

\section{Imagen 5: Propuesta trayecto didáctico para trabajo en AE}

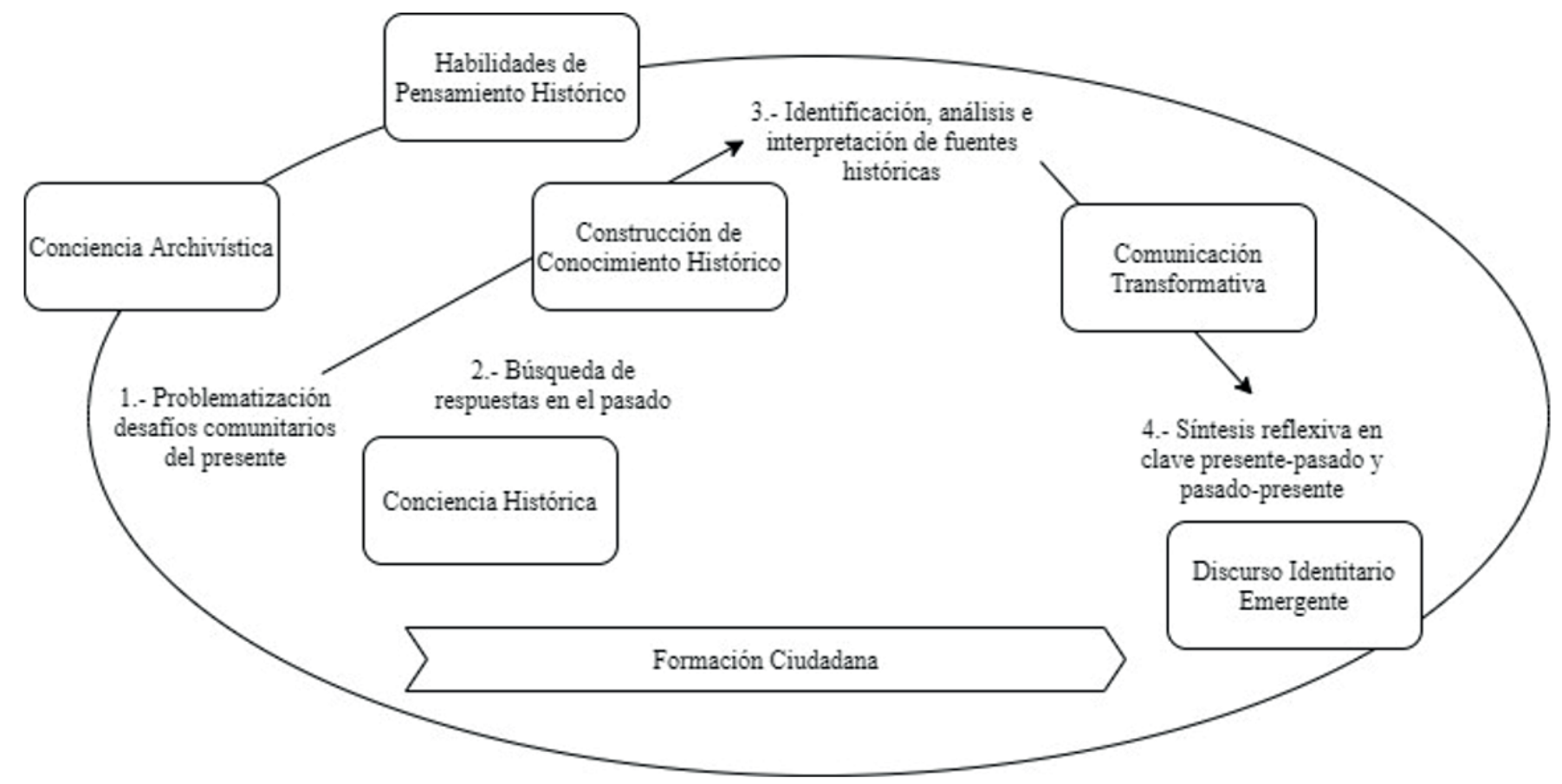


concientizar a la persona porque no sacamos nada con nosotros tener la información guardada y de que nos sirva solamente a nosotros. La idea es que también la comunidad lo sepa y que también la comunidad tome conciencia de esto. Porque si no, por ejemplo, más porque hay una sección de niñas, esa sección de niñas tiene una historia detrás. Y esa historia se convirtió en lo que ahora [...]. Entonces yo creo que es la conciencia que nosotros generamos los estudiantes. (Estudiante, comunicación personal, 2018).

Con lo cual se posibilita la articulación con la construcción de un saber ciudadano para la comunidad educativa y que tiene como fin la socialización del trabajo realizado.

\section{2.- Búsqueda de respuestas en el pasado: Investigar} acerca de las vivencias en el pasado de sujetos de sus comunidades que permitan la construcción de conciencia histórica al sacar del olvido coyunturas relevantes a partir de la elaboración preguntas y objetivos de investigación. Esto posibilita que se desarrollen preguntas en función del por qué, lo que va a permitir a los estudiantes luego dar explicaciones causales del objeto histórico a indagar. Se recomienda que sean 3 o 4 preguntas, las que permitirán estructurar los objetivos y estrategias de recolección de información, tal como señaló uno de los estudiantes en un trabajo donde las fuentes del AE se socializaron vía un "Centro de Interpretación Museal":

Todas estas preguntas, algunas fotografías de todo lo que fue el proceso, se quiere dar a entender cómo era en ese tiempo y cómo se trataba este tema. Cuál era el impacto social que tenía. Entonces nuestro objetivo como centro de información, de interpretación perdón, llegar a la gente de forma más directa para que ellos se interiorizarán en lo que era el trabajo y no fuera, sea algo pasajero como una disertación o algo donde toman más representación lo hablado que lo observado. (Estudiante, comunicación personal, 2018).

De esta forma, teniendo en la centralidad esta búsqueda de respuestas problematizadas, las fuentes del AE a utilizar serán trabajadas con una óptica social llena de sentido para los estudiantes.

3.- Identificación, análisis e interpretación de las fuentes históricas: Buscar y seleccionar fuentes históricas y/o construirlas con metodología de historia oral dependiendo del tema de investigación, de tal forma de que los estudiantes puedan desarrollar conocimiento a partir de la adquisición de herramientas teórico-metodológicas y el fomentar las habilidades de pensamiento histórico como la interpretación y la imaginación histórica (empatía, contextualización y pensamiento crítico).

La búsqueda en el pasado a partir del trabajo con las fuentes desde una metodología activa de los estudiantes como investigadores y constructores de conocimiento, les permitió la "lectura de los documentos", en clave de cuestionar su presente en función de lo hallado en el pasado de su comunidad:

Esto igual da a conocer cómo era el pasado, cómo se interpretaba al resto de la gente y 
cómo igual es como se trataba de ayudar, o sea, igual esto igual puede cambiar cierto pensamiento ante la generación menor que está en el liceo. (Estudiante, comunicación personal, 2018).

Es importante destacar que la conciencia histórica irradia todas las fases, por lo que este ejercicio retrospectivo con las fuentes del AE, permite a los estudiantes posicionarse mediante lo que será el siguiente paso.

4.- Síntesis reflexiva en clave presente-pasado y pasado-presente: Elaborar un espacio de Archivo físico o digital, con cuyas fuentes se logre responder de forma explicativa la pregunta inicial, permitiendo la construcción significativa por parte de los estudiantes de una representación histórica que sea reflexiva entendiendo su presente por el pasado de su comunidad y el pasado por lo que ellos viven en su presente, es decir, teniendo en cuenta categorías del tiempo histórico tales como (cambios y continuidades, vinculación pasado-presente-futuros, duraciones temporales, etc.), posibilitan que puedan hipotetizar, por ejemplo una idea como la siguiente:

[...] la gran parte del liceo no conoce la historia, sino que simplemente viven su presente y no se enfocan en la historia. Y tampoco no hay nadie que les diga la historia y que les diga que esto pasó en tal año. Ahí por lo menos en mi opinión personal me encanta sacar a luz historias que antes habían pasado y que nadie se había pasado, nadie había percatado... (Estudiante, comunicación personal, 2018).
Esta problematización de su identidad en tanto estudiantes de una comunidad educativa que posee una historia, permite una triple dimensión de aprendizajes, por una parte la Conciencia Archivística al vivenciar la importancia del resguardar, conservar y difundir vestigios documentales del pasado para ser problematizados desde el presente. En segundo lugar, el pensar históricamente, como señala Santisteban (2010), permite a los estudiantes "pensar en el tiempo, desplazarse mentalmente en el tiempo y tener conciencia de la temporalidad, para ir construyendo una conciencia histórica que relacione pasado con presente y se dirija al futuro" (2010:39), desarrollando una serie de habilidades para la construcción de una ciudadanía crítica con la que puedan levantar discursos identitarios emergentes desde ellos mismos como sujetos históricos estudiantiles para su comunidad educativa:

Que prácticamente [un hecho] se quedó en el olvido. Que todo este afán por velar por las clases sociales más vulnerables se perdió po. Entonces nosotros, ehh creo yo que nosotros, perdón, cumplimos con el requisito de ser ciudadanos peligrosos porque rompimos un paradigma que no se pensaba, que ya no existía. Y lo volvimos a traer al presente de la noche a la mañana como se dice. Sin ningún aviso previo, solamente nos instalamos y dimos a conocer una actividad que estaba olvidada. (Estudiante, comunicación personal, 2018).

Por ende, la síntesis reflexiva, idealmente debe tener un impacto a la comunidad utilizando estrategias de intervención dentro del espacio 
público de la escuela u otras formas de difusión virtuales que los mismos estudiantes decidan.

\section{Palabras finales}

Chile es un territorio en donde la archivística ha ganado terreno no solo por la ejecución de programas o eventos académicos, sino que también a través de la voluntad de instituciones, organizaciones de la sociedad civil e individuos que se han encargado de desarrollar planes de trabajo y proyectos que han otorgado notoriedad al panorama archivístico chileno, en cuyo caso los AE se configuran como una oportunidad para desarrollar actividades educativas desde una didáctica para la Ciudadanía Democrática.

En ese sentido, concebir la Formación Ciudadana desde una perspectiva transversal, diversa y como Derecho social para nuestros/as estudiantes, permite el desarrollo de actividades que los posicionen no como ciudadanos/as para mañana, sino para el aquí y ahora. El co-construir iniciativas de AE permite una redistribución del poder en el proceso de enseñanza-aprendizaje, dado que la toma de decisiones, temáticas y formas de abordar los contenidos, son consensuados entre docentes y estudiantes. De esta manera horizontalizamos no solo desde los contenidos curriculares las relaciones pedagógicas, sino además desde las habilidades y las actitudes dentro del proceso educativo donde los/as estudiantes en tanto ciudadanos/as, desarrollan y ejercen su Derecho a la Información a través de sus propios AE para la construcción de memoria histórica y prácticas democráticas.
Concebir desde este enfoque didáctico planteado, enriquece los aprendizajes, puesto que aborda el desarrollo de pensamiento crítico, el estudio de temas controversiales, fomenta elementos de pedagogía de la memoria y la construcción de discursos identitarios desde y para los/as estudiantes. Por lo que las comunidades educativas se vuelven sujeto y objeto de su auto reconocimiento en tanto sujetos/as históricos/as, tal como no señaló uno de los estudiantes:

Considero que es una forma nueva, no sé si nueva, sino que novedosa para el espacio social en el que estamos. De romper con el paradigma, que en este caso como ya he dicho antes, es la conciencia social. De cómo se trata, que al final es rememorar un hecho de los años treinta (Estudiante, comunicación personal, 2018).

Este motivo hace relevante señalar la oportunidad de diálogo intergeneracional, dado que los/ as estudiantes actuales, al buscar respuestas en el pasado, se vinculan con las experiencias y aprendizajes de otras generaciones, pero en clave proyectiva. Esta forma de aprendizaje activo y comunitario, debe ser posicionado dentro de las Escuelas, pues fomentar instancias desde un sentido amplio de aula con una didáctica para la autonomía, permite potenciar las habilidades cognitivo-prácticas desde el pensamiento crítico "capaz de pensar [y pensarse], sentir y actuar en su sociedad y en su mundo" (Pagès, 1998, p. 164) mediante el uso y desarrollo de capacidades sobre el cómo abordar los procesos sociales en los que se ven envueltas sus comunidades, de forma conjunta y colaborativa con sus docentes. 
Cabe destacar finalmente que para desarrollar iniciativas de $\mathrm{AE}$ no existe una gran fórmula concreta, sino re-contextualización a los determinados contextos educativos, por lo que visibilizar experiencias que nutran el interés y ganas de levantar este tipo de instancias es algo clave. Quedan muchos desafíos para la sistematización y problematización metodológica que pueda servir de insumo para las distintas comunidades, sobre el cómo organizar, preservar, investigar y difundir su patrimonio documental, por lo que resulta importante seguir esta línea de investigación.

\section{Referencias citadas}

Andaur, G. (2015): "Archivos comunitarios en Santiago de chile: Características generales y reflexiones sobre su estudio", XI Congresso de arquivologia do mercosul arquivos, entre tradição e modernidade São Paulo.

Bianculli, K. Suárez, S. Aguilera, R. y Madroñal, C. (2018): “Los Archivos Escolares: pequeños grandes mundos de conocimiento. Una mirada sobre la producción colaborativa del conocimiento y el patrimonio cultural en la era digital", en Simposio Argentino sobre Tecnologúa y Sociedad, Buenos Aires.

Bravo, L. (2016): “Desde dónde (re) pensar la formación ciudadana hoy”, Docencia, 58, pp. $72-84$.

Cook, T. (2010): "Panoramas del pasado: archiveros, historiadores y combates por la memoria”, TABULA, 13, pp. $153-166$.

Chile. Ley 20.911, crea el Plan de Formación Ciudadana para los establecimientos educacionales reconocidos por el Estado, Ley Chile, 02 de abril de 2016, Disponible en: https://www.bcn.cl/leychile/navegar?idNor$\mathrm{ma}=1088963$

Chile. Ley 20.529, crea el Sistema Nacional de Aseguramiento de la Calidad de la Educación Parvularia, Básica y Media y su fiscalización, 27 de agosto de 2011, Disponible en: https://www.bcn.cl/leychile/navegar?idNorma=1028635

Heredia, A. (1991): "Archivística General. Teoría y Práctica", Archivística, Servicio de Publicaciones de la Diputación de Sevilla, pp. 25-56.

Jímenez, M., L. Bravo y L. Osandón (2013): “La construcción de la ciudadanía contemporánea: nuevas posibilidades para la enseñanza de la historia”, en: I. Muñoz y L. Osandón, eds., La didáctica de la historia y la formación de ciudadanos en el mundo actual, 1st ed. Santiago, Dibam, pp. 139- 155.

Jiménez, M. (2017): “Didáctica de la Ciudadanía” [Material de aula], Universidad Alberto Hurtado, Santiago.

Latorre, A. (2005): La investigación-acción. Conocer y cambiar la práctica educativa, España, Ed. Graó, $3^{\circ}$ edición.

Lodolini, E. (1993): "Investigación sobre la naturaleza y definición del Archivo", Archivística: principios y problemas, Asociación Española de Archiveros, Bibliotecarios, Museólogos y Documentalistas (ANABAD), pp. 125-151.

Lodolini, E. (1995): “El Archivo del Ayer al Mañana”, Boletín de la Asociación Española de Archiveros, Bibliotecarios, Museólogos y Documentalistas (ANABAD), 1, pp. 39-50. 
Mayorga, R, M. Vialy R. Sandoval (2019): Presentación Dossier "Archivos y patrimonio histórico escolar: memoria, pedagogía e investigación", Cuadernos chilenos de Historia de la Educación, 12, pp. 2-5.

Mineduc (2016): Orientaciones para la elaboración del Plan de Formación Ciudadana. Disponible en: https://www.mineduc.cl/wp-content/uploads/sites/19/2016/04/OrientacionesPFC.pdf

Mineduc (2020a): Programa de estudio Comprensión Histórica del Presente. Disponible en: https://www. curriculumnacional.cl/portal/Diferenciado-Humanista-Cientifico/Historia-geografia-y-ciencias-sociales/ Comprension-historica-del-presente/\#objetivos1

Mineduc (2020b): Programa de estudio Educación Ciudadana. Disponible en: https://www.curriculumnacional. cl/portal/Formacion-General/Educacion-ciudadana/ Educacion-ciudadana-3-medio/\#objetivos3

Pagès, J. (1998): "La formación del pensamiento social”, en Benejam, P. y J. Pagès, Enseñar y aprender Ciencias Sociales, Geografía e Historia en la educación secundaria, Barcelona: ICE/Horsori, pp. 152- 164.

Ross, E. W. y Vinson, K. D. (2012): "La educación para una ciudadanía peligrosa”, Revista Enseñanza de las Ciencias Sociales, 11, pp. 73-86.

Sampieri, R., P, Baptista y C. Fernández (2014): Metodología de la Investigación, México, McGRAW-HILL, 6ta edición.

Santisteban, A. (2010) "La formación de competencias de pensamiento histórico”. En Clío \& Asociados (14), 34-56, Clío y Asociados, 14, pp. 34-56.. Disponible en: http://www.memoria.fahce.unlp.edu.ar/art_revistas/ pr.4019/pr.4019.pdf

Soneira, A. (2006): "La teoría fundamentada en los datos (Grounded Theory) de Glaser y Strauss", en I. Vasilachis, ed., Estrategias deinvestigación cualitativa, Madrid, Gedisa, pp. 1533 - 173

Zuñiga, C. y Cárdenas, P. (2019): "Buenas prácticas de ciudadanía en el marco de la Ley de Formación Ciudadana, el pasado 14 de marzo en el Auditorio de la Facultad de Educación UC", en Prácticas sobresalientes en Formación Ciudadana: evidencias de profesores destacados. Proyecto FONDECYT de Iniciación $\mathrm{N}^{\circ} 11150165$. Disponible en: http://ceppe.uc.cl/images/ Presentaciones_Notas/Zuniga_Cardenas.pdf 